\title{
Quality of Life of Patients with Hormone Refractory Prostate Cancer After Gene Therapy
}

\author{
Akinobu Gotoh ${ }^{1}$, Shuji Terao ${ }^{1}$ and Toshiro Shirakawa ${ }^{2}$ \\ ${ }^{1}$ Laboratory of Cell and Gene Therapy, Institute for Advanced Medical Sciences, \\ Hyogo College of Medicine, Nishinomiya, \\ 2 International Center for Medical Research and Treatment, Kobe University \\ School of Medicine, Kobe, \\ Japan
}

\section{Introduction}

Bone metastasis in cancer patients is observed with high clinical frequency and is associated not only with cancer pain but also with pathological fractures, restriction of daily activities, and other skeletal-related events (SRE). It is therefore an issue with a very decisive effect on subsequent quality of life (QOL) (Chow \& Bottomley, 2009). The development of new therapies for bone metastasis in recent years has allowed progress from the use of narcotic formulations, typical of which are the conventional opioid drugs, to treatments focused on 'bone health' which apply a multidisciplinary approach combining use for instance of high-strength bisphosphonate preparations and radionuclide therapy (Satoh et al., 2009). In prostate cancer and breast cancer, which have very high incidence of bone metastasis, there are many cases where survival of a number of years can be expected even at the stage of the illness where this kind of metastasis is present, and the therapeutic strategy needs to take account of QOL.

Each year thousands of men are diagnosed with prostate cancer; it is estimated that about 192,280 men will be diagnosed with this disease in 2009 (American Cancer Society, 2008). In advanced disease, androgen-deprivation therapy remains the best treatment. After a median response of 12 to 24 months, patients develop progressive hormone-refractory prostate cancer (HRPC) (Carroll et al., 2002, Chodak et al., 2002). Therefore, the development of new therapies with a survival advantage and greater antitumor effects is necessary. Gene therapy is a good candidate among new therapies.

The phase I clinical trial of the combination therapy of osteocalcin promoter-driven herpessimplex-virus thymidine kinase (Ad-OC-TK) and valacyclovir (VAL) for the treatment of HRPC has been conducted previously at the University of Virginia (Kubo et al., 2003). Between August 2003 and March 2006, a phase I/II dose-escalating clinical trial of intratumoral injections of Ad-OC-TK was performed on 6 patients with HRPC in order to assess the potential toxicity and therapeutic effects of this treatment at Kobe University Hospital (Hinata et al., 2006, Shirakawa et al., 2007). In clinical oncological trials, the therapeutic effects on the tumor volume and the serum levels of tumor markers are frequently used as the primary therapeutic indices. However, recently, the assessment of QOL has become an important therapeutic index. Since prostate cancer generally has a 
protracted natural history, it is particularly important for patients with HRPC to receive therapies that preserve or improve QOL, delay the progression of disease caused by severe pain, and provide quality-adjusted clinical benefits (Ho et al., 2006).

Among the QOL assessment systems developed so far are the European Organization for Research and Treatment of Cancer (EORTC), Quality of Life Questionnaire-Core 30 (QLQC30) (Aaronson et al., 1993), Functional Assessment of Cancer Therapy scale-General (FACT-G) (Cella et al., 1993) and MOS Short-Form 36-Item Health Survey (SF-36) (Ware \& Sherbourne, 1992) systems, which have been translated and tested for use in a wide range of languages. Modules for quality of life assessment in specific diseases have also been developed and applied clinically, for instance EORTC-QLQ-LC13 (Bergman et al., 1994) and FACT-L (Cella et al., 1995) for lung cancer, EORTC-QLQ-BR23 (Sprangers et al., 1996) and FACT-B (Brady et al., 1997) for breast cancer, and European Organization for Research and Treatment of Cancer Quality of Life Questionnaire's prostate cancer-specific module (EORTC QLQ-PR25) (da Silva et al., 1993) and FACT-P (Esper et al., 1997) for prostate cancer. However, no QOL module had so far been developed for bone metastasis patients. We designed a questionnaire, formulated with reference to the EORTC-QLQ- PR25 assessment system. This is the first study, the short-term QOL and bodily pain of patients with HRPC who were undergoing a clinical trial involving the direct administration of the combination of Ad-OC-TK gene therapy plus VAL intra-lesionally in men with metastatic prostate cancer was evaluated.

\section{Patients and methods}

\subsection{Eligibility criteria}

The eligibility criteria included the presence of metastatic or locally recurrent prostate cancer, histologically confirmed adenocarcinoma, and confirmed disease progression despite surgery, radiation, androgen blockage, and/or conventional chemotherapy. Patients who were receiving androgen deprivation therapy including anti-androgen therapy underwent an at least 4-week anti-androgen withdrawal period before enrollment to exclude possible effects of anti-androgen withdrawal syndrome (Scher and Kelly, 1993). Luteinizing hormone-releasing hormone(LH-RH) agonist was continued during the antiandrogen withdrawal period and throughout the study. Further, each patient included must have had a lesion measurable by an imaging examination, and a single selected lesion had to be amenable to intralesional injection of the therapeutic agent.

\subsection{Vector and valacyclovir}

The replication-deficient adenoviral vector belongs to subgenus $C$, serotype 5 (Ad5) with the HSV-tk gene under the control of a tumor-restrictive OC promoter in the area of the excised E1 region. Ad-OC-TK was constructed via homologous recombination vector, as described previously (Ko et al., 1996). The Good Manufacturing Practice (GMP)-grade adenoviral vector (Lusky, 2005) was manufactured and characterized extensively by Molecular Medicine Bioservices (Carlsbad, CA) before clinical application. The oral antiviral agent valacyclovir (Valtrex; GlaxoSmithKline, Tokyo, Japan) has been approved in Japan for several indications, including treatment for herpes zoster and genital herpes. We used the worldwide-approved valacyclovir dosage of $1 \mathrm{~g}$, three times daily (Tyring et al., 2000) in our clinical trial. 


\subsection{Study design}

Six patients were divided into the low-dose and high-dose viral vector group (3 patients at each dose level). Ad-OC-TK was injected directly into the localized recurrent tumor or the bone metastatic lesion under the guidance of transrectal ultrasonography (TRUS) or computed tomography (CT). Only one index lesion per patient was selected, typically that representing the largest metastasis, causing severe bone pain at the metastatic site, or preventing urination by local recurrent tumor. The doses used were $2.5 \times 10^{9}$ (low-dose group: 3 patients) and $2.5 \times 10^{10}$ (high-dose group: 3 patients) plaque-forming units (PFU) on Day 1 and Day 8 . The patients were given $1 \mathrm{~g}$ of VAL three times daily for 21 days.

\subsection{Assessment of the short-term QOL and bodily pain}

Since there was no questionnaire for assessing the short-term QOL in patients receiving gene therapy for HRPC, we created a questionnaire (Table 1) (Terao, 2009) was created based on the EORTC QLQ-PR25 which is widely used to assess QOL in patients of prostate cancer. The questionnaire comprised scores evaluating role limitations in work (2 items), physical or social functioning (4 items), interpersonal relationships (2 items), emotional functioning (3 items), and sleep/vitality (2 items) as well as the global health status (2 items). A higher median value obtained on the symptom scale indicated a higher number of symptoms or problems. All the patients answered the questionnaire by themselves on days 0 (pretreatment), 14 and 28. The patients indicated how true a statement had been for them over the past 14 days using a 4-point scale as follows: 0 , not at all; 1 , a little bit; 2, quite a bit; 3 , very much. All items were assigned equal weightage. Further, all the patients scored themselves on a visual analogue scale (VAS) with regard to their bodily pain on day 0,14 and 28 as follows: $0 \%$, they did not experience any bodily pain; $100 \%$, they experienced severe bodily pain (EuroQol, 1990).

Global health status

-How much influence in your health?

-How much influence in your life?

Role limitation of work

-How much influence in your home works such as clean, shopping and so on ?

-How much influence in the work and the daily activity out of home?

Role limitation of physical or social

- How much influence to move your body such as stroll, running, sports and so on?

-How much influence to use a bus, a car, train, an airplane and so on?

- How much influence in the relationship having to do with the world?

- How much influence to call or meet your friends?

Personal relationship

-How much influence in the relation with your companion and partner?

-How much influence in the life with the family?

Emotional

-Did you felt depress?

-Did you felt anxiety and became nervous?

-Did you became miserable?

Sleep /Vitality

-How much influence in the dormancy?

-Did you felt fatigue?

Table 1. Questionnaire. Answer questions from 0-3 (0: not at all; 1: a little bit; 2: quite a bit; 3: very much). 


\subsection{Correlation of the change of PSA with QOL and bodily pain}

The correlation of serum prostate-specific antigen (PSA) levels with short-term total QOL scores and bodily pain was assessed by measuring the rate of change (\%) of the serum PSA levels, the short-term total QOL scores, and bodily pain from day 0 to day 28; these values were expressed as $\triangle \mathrm{PSA}, \triangle \mathrm{QOL}$, and $\triangle$ Pain, respectively (Terao et al., 2009).

\subsection{Statistical analysis}

The short-term QOL scores of patients (nonparametric data) were analyzed by Friedman one-way ANOVA. The Spearman test was used to analyze the correlation of $\triangle$ PSA with $\triangle$ QOL and $\triangle$ Pain. $P<0.05$ was considered to be statistically significant.

\section{Results}

\subsection{Patient characteristics}

The mean age of the patients was 68 years (range, 63-77 years). The mean pretreatment serum PSA level was $222.7 \mathrm{ng} / \mathrm{mL}$ (range, 46.6-455.5 ng/mL). Three patients $(50 \%$ ) had been diagnosed with poorly differentiated adenocarcinoma and the other $3(50 \%)$ had been diagnosed with moderately differentiated adenocarcinoma. All the patients had multiple bone metastatic lesions. In addition, 2 patients (33.3 \%) had locally recurrent lesions. Two patients $(33.3 \%)$ had undergone radical prostatectomy in the past and all the patients had been treated with androgen-deprivation therapy.

\subsection{Short-term QOL and bodily pain}

All 6 patients answered the questionnaires on days 0, 14 and 28. Table 2 (Terao, 2009) shows the short-term QOL scores of role limitations in work, physical or social functioning, interpersonal relationships, emotional functioning and sleep/vitality along with a total score of all items as well as the global health status.).

\begin{tabular}{|c|c|c|c|}
\hline Items & pretreatment & Day 14 & Day 28 \\
\hline \multicolumn{4}{|l|}{ QOL score } \\
\hline Global health status & $6.0(5-7)$ & $5.5(5-6)$ & $5.0(4-6)^{*}$ \\
\hline Role limitations in work & $6.0(5-6)$ & $4.5(5-6)$ & $4.0(4-6)^{* \star *}$ \\
\hline Physical or social functioning & $11.0(9-12)$ & $8.5(8-10)^{*}$ & $8.0(6-10)^{*}$ \\
\hline Interpersonal relationships & $6.0(4-6)$ & $5.0(3-6)^{*}$ & $4.0(3-6)^{* *}$ \\
\hline Emotional functioning & $7.5(7-9)$ & $7.0(5-7)$ & $5.0(5-7)^{* *}$ \\
\hline Sleep /Vitality & $5.0(4-6)$ & $4.5(4-6)$ & $4.0(4-5) * \star$ \\
\hline Total score & $42.0(35-54)$ & $34.0(31-39)$ & $30.5(27-37)^{* * *}$ \\
\hline Bodily pain (\%) & $52.5(10-70)$ & $35.0(5-50)$ & $25.0(5-50)^{* * *}$ \\
\hline
\end{tabular}

Data is expressed as median (range). ${ }^{*} \mathrm{p}<0.05,{ }^{*-k} \mathrm{p}<0.01$

Table 2. Scores of QOL and percent of bodily pain. 
As compared with day 0 , the scores for the global health status $(P<0.05)$, interpersonal relationships $(P<0.01)$, emotional functioning $(P<0.01)$ and sleep/vitality $(P<0.01)$ were significantly improved on day 28. Moreover, significant improvements in the short-term QOL scores were demonstrated with respect to the role imitations in work (day 14, $P<0.05$; day $28, P<0.05$ ) and physical or social functioning (day $14, P<0.05$; day $28, P<0.01$ ). As compared with day 0 , the total score of all items was also significantly improved on day 28 $(P<0.01)$. Further, as compared with day 0 , the bodily pain was significantly reduced on day $28(P<0.01)$.

\subsection{Correlation of $\triangle \mathrm{PSA}$ with $\triangle \mathrm{QOL}$ and $\triangle \mathrm{Pain}$}

Figure 1 shows the changes in the serum PSA levels of all patients from a period of 8 weeks before $(-8 \mathrm{w})$ to 4 weeks after $(4 \mathrm{w})$ the initial vector injection. The correlation of the serum PSA levels with the short-term total QOL scores and bodily pain is shown in Figure 2(A) and Figure 2(B), respectively. A significant correlation was observed between $\triangle$ PSA and $\triangle$ QOL $(P<0.05)$; however, it was not observed between $\triangle$ PSA and $\triangle$ Pain (Terao, 2009).

\section{Discussion}

Bone metastasis is found in 70 to $85 \%$ of autopsy cases in malignant tumor patients, and the bone is a potential site of metastasis in all cancerous tumors (Tubiana-Hulin, 1991). In recent years, the use of third-generation bisphosphonates and the progress of multidisciplinary approaches combining radiotherapy, chemotherapy, and other treatments has led to improvement in SREs and prolongation of survival in advanced cancers (Saad et al., 2002), so that long-term survival can be expected in prostate cancer, breast cancer, multiple myeloma, and other conditions even at the stage where bone metastasis is present. In conjunction with this progress, a strategy and response focused on long-term quality of life is required beginning from the initial stage of treatment. This is now coming to be seen as an important item for assessment when developing novel therapies. The present study consisted of a quality of life assessment in prostate cancer patients following the novel treatment of gene therapy.

QOL instruments, including EORTC QLQ-PR25, have been applied in several therapeutic trials of prostate cancer (Ho et al., 2006, Cella et al., 2006, Canil et al., 2005). The items in these questionnaires include questions regarding urinary conditions, for example pollakiuria, dysuria, and macrohematuria. Further, these questionnaires are employed for patients of prostate cancer in various stages. In this study, however, patients who have undergone various treatments-including radical prostatectomy, androgen-deprivation therapy and/or conventional chemotherapy-prior to gene therapy were targeted. Further, in 4 patients, the lesions that were injected with the vector were bone metastatic lesions alone. This study aimed at assessing the effect of gene therapy on bodily pain because most patients suffered pain at the site of vector-injected lesion. Moreover, there have been no questionnaires that assess QOL in patients receiving gene therapy for HRPC. Taking the above into consideration, a questionnaire based on EORTC QLQ-PR25 questionnaire (Table 1) was created and bodily pain was assessed by using VAS (Tearo et al., 2009). 


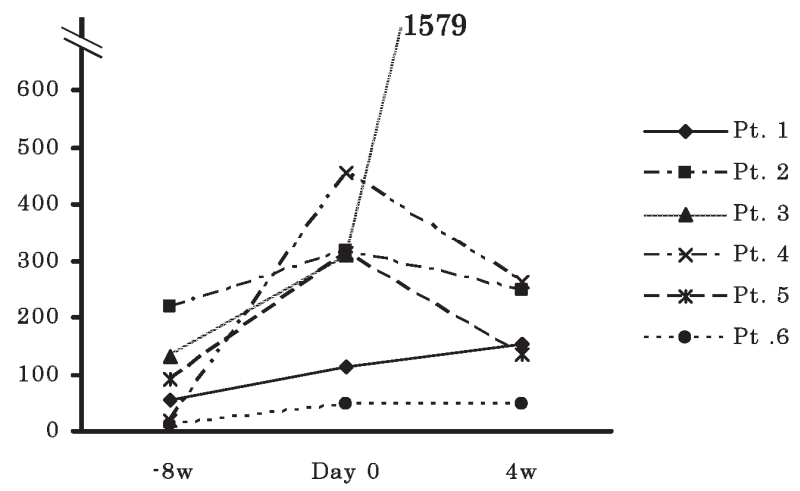

Fig. 1. The change of serum PSA level in all patients from before 8 weeks $(-8 w)$ to after 4 weeks $(4 \mathrm{w})$ of gene therapy.

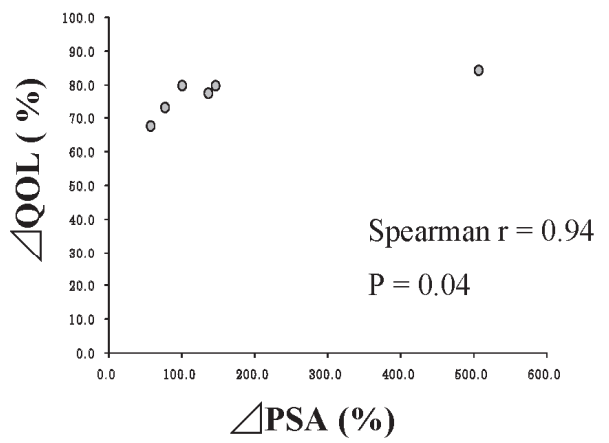

(A)

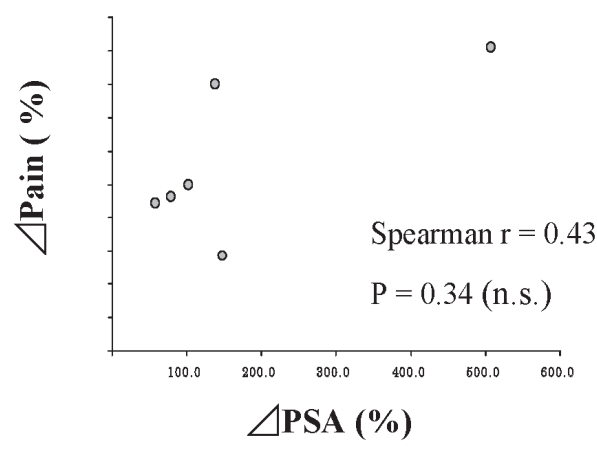

(B)

Fig. 2. (A) The correlation of PSA levels with short-term total QOL scores. (B) The correlation of PSA levels with bodily pain. Both were assessed by measuring the rate of change $(\%)$ of the serum PSA levels, the short-term total QOL scores, and bodily pain from day 0 to day 28 ; these values were expressed as $\triangle \mathrm{PSA}, \triangle \mathrm{QOL}$, and $\triangle \mathrm{Pain}$, respectively. 
Recently QOL is increasingly being recognized as a measure for assessing outcomes of therapeutic trials. Further, the assessment of the clinical effects of therapy on QOL is indispensable to the evaluation of therapeutic trials (Fayers \& Bottomley, 2005). In several therapeutic trials for prostate cancer, the assessment of patients' QOL is incorporated as a therapeutic index. In this study, it was demonstrated that Ad-OC-TK plus VAL treatment significantly improved the short-term QOL scores and the total score using the modified questionnaire statistically correlated between $\triangle \mathrm{PSA}$ and $\triangle \mathrm{QOL}$ (Figure 2(A)). Therefore, although it will be necessary to compare the changes in QOL scores using the modified questionnaire to the changes in QOL scores using other typical questionnaires that are used for patients of HRPC, it is considered that the QOL of patients receiving Ad-OC-TK plus VAL treatment is worth assessing as a therapeutic index. Further, significant improvements in bodily pain were also observed. It is suggested that the clinical improvements may contribute to a significant improvement in bodily pain. Since the tumors of these patients displayed disease progression despite surgery, radiation, androgen-deprivation therapy and/or conventional chemotherapy, the serum PSA levels of all patients were very high prior to gene therapy. Following gene therapy, the serum PSA levels decreased in 2 patients, no change was observed in 1 patient and they were slightly increased in 2 patients (Figure 2(A)). Further, the induction of cell apoptosis in the vector-injected lesions of 5 patients examined by anti-ssDNA antibody staining on day 28 was also observed. However, a correlation between $\triangle \mathrm{PSA}$ and $\triangle \mathrm{Pain}$ was not demonstrated in spite of the clinical effects observed in the vector-injected lesion (Figure 2(B)). In this study, the Ad-OC-TK vector was injected directly into one single localized recurrent tumor or bone metastatic lesion despite of the fact that all the patients had multiple bone metastatic lesions. Especially in the case of patient 3 , the pain in the non-vector injected lesions increased on day 28 . This fact might be one reason that a correlation between $\triangle \mathrm{PSA}$ and $\triangle$ Pain was not demonstrated (Terao et al., 2009).

Pain, which has been called the fifth vital sign, has come to be recognized as a crucial factor in assessing patient condition in the clinical environment. At the same time, however, pain is a subjective phenomenon, which makes it difficult to gain an accurate picture of a patient's pain. It will be important in future to perform diagnosis using more appropriate assessment methods. In the assessment of the efficacy of gene therapy as a novel cancer therapy, assessment methods emphasizing QOL are likely to receive important attention in the future. Since it has been found that the subjective sense of physical well-being in a large proportion of Japanese patients is centered not on the self but on external objects such as the family or the doctor, it would seem important to assess and select therapies with care, taking into account psychological aspects which patients find difficult to express for themselves, and basing the process on an assessment of the effect of each therapeutic method on the basic QOL.

\section{Conclusion}

It was demonstrated that Ad-OC-TK plus VAL gene therapy improved or preserved the short-term QOL and bodily pain of HRPC patients with metastatic or local recurrent tumors. These assessments may emerge as the new therapeutic indices of gene therapy for HRPC. 


\section{Acknowledgment}

We thank all the patients for their participation in this study. This research was supported as a Japanese Research Project, Grant-in-aid for Scientific Research (A) : project 1420763.

\section{References}

Aaronson, N.K., Ahmedzai, S., \& Bergman, B., et al. (1993). The European Organization for Research and Treatment of Cancer QLQ-C30; a quality-of-life instrument for use in international clinical trials in oncology. J Natl Cancer Inst, 85, 365376.

American Cancer Society. (2008). Cancer Facts and Figures, 2008. Atlanta, GA, USA

Bergman, B., Aaronson, N.K. \& Ahmedzai, S. (1994). The EORTC QLQ-LC13: a modular supplement to the EORTC Core Quality of Life Questionnaire (QLQ-C30) for use in lung cancer clinical trials. EORTC Study Group on Quality of Life. Eur J Cancer, 30A, 635-642.

Brady, M.J., Cella, D.F., \& Mo, F. (1997). Reliability and validity of the Functional Assessment of Cancer Therapy-Breast quality-of-life instrument. J Clin Oncol, 15, 974-986.

Canil, C.M., Moore, M.J., \& Seymour, L. et al. (2005). Randomized phase II study of two doses of gefitinib in hormone-refractory prostate cancer: a trial of the National Cancer Institute of Canada-Clinical Trials Group. J Clin Oncol. 23, 455460.

Carroll, P.R., Kantoff, P.W., \& Balk, S.P., et al. (2002). Second International Conference on Newer Approaches to Androgen Deprivation Therapy (ADT) in Prostate Cancer overview consensus statement-newer approaches to androgen deprivation therapy in prostate cancer. Urology. 60, 1-6.

Cella, D., Tulsky, D.S., \& Gray, G., et al. (1993). The Functional Assessment of Cancer Therapy scale: development and validation of the general measure. J Clin Oncol 11, 570-579.

Cella, D., Bonomi, A.E., \& Lloyd, S.R. (1995). Reliability and validity of the Functional Assessment of Cancer Therapy-Lung (FACT-L) quality of life instrument. Lung Cancer, 12, 199-220.

Cella, D., Petrylak, D., \& Mulani, P., et al. (2006). Role of quality of life in men with metastatic hormone-refractory prostate cancer: how does atrasentan influence quality of life? Eur Urol. 49, 781-789.

Chodak, G.W., Keane, T., \& Klotz, L., et al. (2002). Critical evaluation of hormonal therapy for carcinoma of the prostate. Urology. 60, 201-208.

Chow, E., \& Bottomley, A. (2009). Understanding the EORTCQLQ-BM22, the module for patients with bone metastases. Expert rev Pharmacoecon Outcomes Res, 9, 461465.

da Silva, F.C., Reis, E., Costa, T., \& Denis, L., et al. (1993). Quality of life in patients with prostatic cancer. A feasibility study. The Members of Quality of Life Committee of the EORTC Genitourinary Group. Cancer, 71, 1138-1142. 
Esper, P., Mo, F., \& Chodak, G., et al. (1997). Measuring QOL in men with prostate cancer using the FACT-P instrument. Urology, 50, 920-928.

EuroQol. (1990). A new facility for the measurement of health-related quality of life. The EuroQol Group. Health Policy, 16, 199-208.

Fayers, P., \& Bottomley, A. (2002). EORTC Quality of Life Group; Quality of Life Unit. Quality of life research within the EORTC-The EORTC QLQ-C30. European Organisation for Research and Treatment of Cancer. Eur J Cancer, 38, 125133.

Hinata, N., Shirakawa, T., \& Gotoh, A., et al. (2006). Progress report on phase I/II clinical trial of Ad-OC-TK plus VAL therapy for metastatic or locally recurrent prostate cancer: Initial experience at Kobe University. Int J Urol, 13, 834-837.

Ho, S., Wook, J., \& Jong, J. (2006). Targeted-cryosurgical ablation of the prostate with androgen deprivation therapy: quality of life in high-risk prostate cancer patients. Asian J Androl, 8, 629-636.

Ko, S.C., Cheon, J., \& Chung, L.K. (1996). Osteocalcin promoter-based toxic gene therapy for the treatment of osteosarcoma in experimental models. Cancer Res, 56, 46144619.

Kubo, H., Gardner, T.A., \& Chung, L.W., et al.(2003). Phase I dose escalation clinical trial of adenovirus vector carrying osteocalcin promoter-driven herpes simplex virus thymidine kinase in localized and metastatic hormone-refractory prostate cancer. Hum Gene Ther, 14, 227-241.

Lusky, M. (2005) Good manufacturing practice production of adenoviral vectors for clinical trials. Hum Gene Ther, 16, 281-291.

Saad, F., Gleason, D.M., \& Murray, R., et al. (2002). A randomized, placebo-controlled trial of zoledronic acid in patients with hormone-refractory metastatic prostate carcinoma. J Natl Cancer Inst, 94, 1458-1468.

Satoh, T., Kimura, M., \& Matsumoto, K., et al. (2009). Single infusion of zoledronic acid to prevent androgen-deprivation therapy-induced bone loss in men with hormonenaïve prostate carcinoma. Cancer, 115, 3468-3474.

Scher, H.I., \& Kelly, W.K. (1993). Flutamide withdrawal syndrome: Its impact on clinical trials in hormone-refractory prostate cancer. J Clin Oncol, 11, 1566-1572.

Shirakawa, T., Terao, S., \& Gotoh, A., et al. (2007). Long-term outcome of phase I / II clinical trial of Ad-OC-TK/VAL gene therapy for hormone-refractory metastatic prostate cancer. Hum Gene Ther, 18, 1225-1232.

Sprangers, M.A., Groenvold, M., \& Arraras, J.I. (1996). The European Organization for Research and Treatment of Cancer breast cancer-specific quality-of-life questionnaire module: first results from a three-country field study. J Clin Oncol, 14, 2756-2768.

Terao, S., Shirakawa, T., \& Gotoh, A., et al. (2009). A pilot study of quality of life of patients with hormone-refractory prostate cancer after gene therapy. Anticancer Res, 29, 1533-1538.

Tubiana-Hulin, M. (1991). Incidence, prevalence and distribution of bone metastases. Bone, 12, S9-10. 
Tyring, S.K., Beutiner, K.R., \& Crooks, R.J., et al. (2000). Antiviral therapy for herpes zoster: Randomized, controlled clinical trial of valacyclovir and famciclovir therapy in immunocompetent patients 50 years and older. Arch Fam Med, 9, 863869.

Ware, J.E. Jr, \& Sherbourne, C.D. (1992). The MOS 36-item short-form health survey (SF-36). I. Conceptual framework and item selection. Med Care, 30, 473-483. 


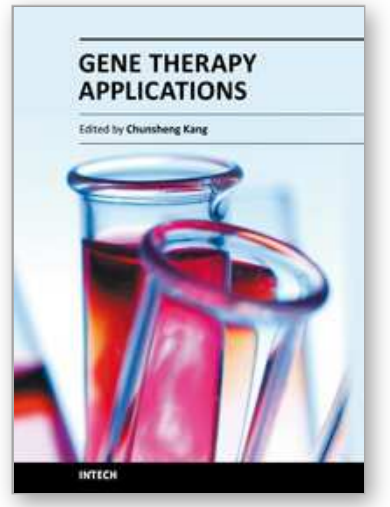

\author{
Gene Therapy Applications \\ Edited by Prof. Chunsheng Kang
}

ISBN 978-953-307-541-9

Hard cover, 492 pages

Publisher InTech

Published online 23, August, 2011

Published in print edition August, 2011

The aim of our book is to provide a detailed discussion of gene therapy application in human diseases. The book brings together major approaches: (1) Gene therapy in blood and vascular system, (2) Gene therapy in orthopedics, (3) Gene therapy in genitourinary system, (4) Gene therapy in other diseases. This source will make clinicians and researchers comfortable with the potential and problems of gene therapy application.

\title{
How to reference
}

In order to correctly reference this scholarly work, feel free to copy and paste the following:

Akinobu Gotoh, Shuji Terao and Toshiro Shirakawa (2011). Quality of Life of Patients with Hormone Refractory Prostate Cancer After Gene Therapy, Gene Therapy Applications, Prof. Chunsheng Kang (Ed.), ISBN: 978953-307-541-9, InTech, Available from: http://www.intechopen.com/books/gene-therapy-applications/qualityof-life-of-patients-with-hormone-refractory-prostate-cancer-after-gene-therapy

\section{INTECH}

open science | open minds

\section{InTech Europe}

University Campus STeP Ri

Slavka Krautzeka 83/A

51000 Rijeka, Croatia

Phone: +385 (51) 770447

Fax: +385 (51) 686166

www.intechopen.com

\section{InTech China}

Unit 405, Office Block, Hotel Equatorial Shanghai

No.65, Yan An Road (West), Shanghai, 200040, China

中国上海市延安西路65号上海国际贵都大饭店办公楼 405 单元

Phone: +86-21-62489820

Fax: +86-21-62489821 
(C) 2011 The Author(s). Licensee IntechOpen. This chapter is distributed under the terms of the Creative Commons Attribution-NonCommercialShareAlike-3.0 License, which permits use, distribution and reproduction for non-commercial purposes, provided the original is properly cited and derivative works building on this content are distributed under the same license. 